\title{
The Stark Ladder and Other One-Dimensional External Field Problems
}

\author{
I. W. Herbst ${ }^{1}$ and J. S. Howland ${ }^{2}$
}

Department of Mathematics, University of Virginia, Charlottesville, VA 22903, USA

\begin{abstract}
For a certain class of analytic potentials $V(x)$, matrix elements of the resolvent of $H_{F}=-d^{2} / d x^{2}+F x+V(x)$ with entire vectors of the translation group have meromorphic continuations from $\operatorname{Im} z>0$ to the whole complex plane. The poles of these continuations are restricted to a discrete set independent of the analytic vectors chosen. Certain random potentials corresponding to an infinite number of particles distributed on the points of a Poisson set lie in this class with probability one as do a large class of periodic potentials.
\end{abstract}

\section{Introduction}

It is believed that when a uniform electric field $F$ is applied to a one-dimensional periodic solid described by

$$
H=-d^{2} / d x^{2}+V(x)
$$

with $V(x)$ periodic of period 1, each band gives rise to an infinite sequence of resonances of fixed imaginary part, located at

$$
E_{n}=E_{0}+n F \quad n=0, \pm 1, \pm 2, \ldots
$$

where $\operatorname{Im} E_{0}<0$. Although the Hamiltonian

$$
H_{F}=-d^{2} / d x^{2}+F x+V(x)
$$

may look rather simple, no rigorous proof of the existence of these resonances has yet been given, to say nothing of the important problem of estimating the lifetime. Attacking the problem from a different point of view, Bentosela [6] has shown, in any number of dimensions, the existence of states $\psi$ such that $e^{-i t H_{F}} \psi$ has a momentum distribution with the momentum nearly periodic in time over many periods $T=2 \pi / F$. 
It is our purpose here to give a framework for the discussion of this problem by means of the translation analyticity technique introduced in [4]. We show that the operator

$$
H_{F}(-i a)=-d^{2} / d x^{2}+F x+V(x-i a)-i a F, \quad a>0
$$

obtained by subjecting $H_{F}$ to a complex translation $i a$ has its essential spectrum contained in $\mathbb{R}-i a F$, provided that $V(z)$ is analytic in a strip $|\operatorname{Im} z|<a_{0}$, with $a<a_{0}$, and satisfies a growth estimate.

This includes a large class of periodic and almost periodic potentials. Once this is accomplished, one has standard machinery with which to discuss resonances $[1,4,5]$. The difficulty is that $V(x-i a)$ is not a relatively compact perturbation of $-d^{2} / d x^{2}+F x$. We get around this by treating $H_{F}(-a)-z$ as a perturbation of a $(z, a)$-dependent operator $B(z, a)$ whose inverse is known more or less explicitly from a WKB approximation.

We have also considered the problem for a class of random potentials on $\mathbb{R}$. Such potentials have been studied widely in recent years as models of unordered materials. For a large class of $V$, the operator $-d^{2} / d x^{2}+V$ has almost surely a pure point spectrum, dense in, say, $[0, \infty)$, if $V$ is positive $[9,10]$ indicating zero conductivity in the $F \rightarrow 0$ and zero temperature limit. The phenomenon is known as Anderson localization $[2,14]$. It seems of interest to consider the case $F \neq 0$. We study a model where identical atoms are placed randomly on the line at the locations of a Poisson ensemble of points. This leads to the potential

$$
V(x)=\sum_{j=-\infty}^{+\infty} u\left(x-X_{j}\right)
$$

where $u(x)$ is the potential due to each atom, and $X_{j}$ is the (random) position of the $j$ th atom. If $u(x)$ satisfies certain assumptions (including integrability and analyticity in a strip), we are able to show that almost surely $V(z)$ is analytic there and

$$
V(z)=0(\log |z|) \text {. }
$$

With this growth estimate, our result on the essential spectrum is shown to be applicable to this case.

We remark that our results obviously still apply if a periodic potential is added to our random potential. We then have a model of impurities in a crystal, the density of which can be varied continuously by changing the parameter in the Poisson distribution. It would be interesting to have an almost sure result on the location of resonances as $F \rightarrow 0$ but we have not studied this problem. It would also be of interest to prove Anderson localization for our model with $F=0$.

Section 2 of this paper is devoted to proving the essential spectrum result for $H_{F}(a)$ discussed above and the results on resonances which follow from it. Here we make assumptions about the potential $V$ which we verify in Section 3 for our random potential.

We use the notations $\mathbb{R}$ for the reals, $\mathbb{C}$ for the complexes, and Ran $L$, Ker $L$ and $\mathscr{D}(L)$ for the range, kernel and domain of an operator $L$. 


\section{Translation Analyticity for $H$}

In this section we will be concerned with the operator $-\frac{d^{2}}{d x^{2}}+x+V_{\lambda}(x)+\lambda$ (we set $F=1$ without loss of generality) where $V_{\lambda}(x)=V(x+\lambda)$ and $\lambda$ is complex. Because $V_{\lambda}$ will be unbounded at infinity even the definition of this operator is not obvious. We will always assume that $V$ obeys the following conditions:

(a) $V$ is analytic in $S_{a_{0}}=\left\{z:|\operatorname{Im} z|<a_{0}\right\}$ where $0<a_{0} \leqq \infty$.

(b) If $0<a<a_{0},|V(z)| \leqq c_{a}\left(1+|z|^{1 / 2-\varepsilon}\right)$ for all $z \in S_{a}$ and for some $\varepsilon>0$.

(c) $V$ is real valued on the real axis.

For $\lambda$ real, it follows from a result of Faris and Lavine [7] that $-\frac{d^{2}}{d x^{2}}+x+V_{\lambda}+\lambda$ is essentially self-adjoint on $C_{0}^{\infty}(\mathbb{R})$. We denote the closure of this operator by $H(\lambda)$ and set $H=H(0)$.

Following Reed and Simon [11, p. 236], we shall define the essential spectrum $\sigma_{\text {ess }}(L)$ of a closed operator $L$ to consist of all points of the spectrum $\sigma(L)$, except for isolated eigenvalues of finite algebraic multiplicity. Thus, the complement of $\sigma_{\text {ess }}(L)$ is the set of $z$ on which $(L-z)^{-1}$ is meromorphic, with finite rank principal parts at each of its poles. The discrete spectrum is defined as $\sigma_{\text {disc }}(L)=$ $\sigma(L) \backslash \sigma_{\text {ess }}(L)$.

For real $a$, let $U(a)$ be the unitary translation operator:

$$
U(a) f(x)=f(x+a) .
$$

The goal of this section is to prove the following result:

2.1 Theorem Assume $V$ satisfies a), b) and c) above. Then

(i) The operator $\left(-\frac{d^{2}}{d x^{2}}+x+\lambda+V_{\lambda}\right) \mid C_{0}^{\infty}(\mathbb{R}), \lambda \in S_{a_{0}}$ is closable. Denote its closure by $H(\lambda)$. For $a \in \mathbb{R}$ we have $U(a) H(\lambda) U(-a)=H(\lambda+a)$.

(ii) For $\lambda \in S_{a_{0}}, H(\lambda)^{*}=H(\bar{\lambda})$

(iii) For $\lambda \in S_{a_{0}}, \sigma_{\text {ess }}(H(\lambda)) \subseteq \mathbb{R}+\lambda$

(iv) If $0<\operatorname{Im} \lambda<a_{0}$,

$$
\sigma_{\text {disc }}(H(\lambda)) \subseteq\{z: 0 \leqq \operatorname{Im} z \leqq \operatorname{Im} \lambda\}
$$

and if $0<\operatorname{Im} \mu \leqq \operatorname{Im} \lambda$, then

$$
\tilde{\sigma}_{\text {disc }}(H(\mu))=\tilde{\sigma}_{\text {disc }}(H(\lambda)) \cap\{z: 0 \leqq \operatorname{Im} z<\operatorname{Im} \mu\}
$$

where $\tilde{\sigma}_{\text {disc }}(H(\lambda))=\sigma_{\text {disc }}(H(\lambda)) \cap\{z: \operatorname{Im} z \neq \operatorname{Im} \lambda\}$. Because of (ii) there is a similar statement for $-a_{0}<\operatorname{Im} \lambda<0$.

(v) $(z-H(\lambda))^{-1}$ is analytic in $\lambda$ for $\lambda \in D_{z}$ where $D_{z}=\left\{\lambda \in S_{a_{0}}: \operatorname{Im} \lambda>\operatorname{Im} z\right.$, $z \notin \sigma(H(\lambda))\}$ and for $\lambda \in E_{z}=\left\{\lambda \in S_{a_{0}}: \operatorname{Im} \lambda<\operatorname{Im} z, z \notin \sigma(H(\lambda))\right\}$.

The following Corollary justifies the consideration of $H(\lambda)$ for complex $\lambda$. Let $H(0)=H$.

2.2 Corollary. Assume $V$ satisfies a), b), c) above. Let $\mathscr{D}$ be the family of all $f \in L^{2}(\mathbb{R})$ 
such that $U(a)$ fhas an $L^{2}$-valued analytic continuation to $|\operatorname{Im} a|<a_{0} \cdot$ Let

$$
\mathscr{R}_{a_{0}}=\bigcup_{0<a<a_{0}} \sigma_{\text {disc }}(H(-i a)) .
$$

Then if $f \in \mathscr{D}$, the function $G(f, z)=\left(f,(z-H)^{-1} f\right)$ has an analytic continuation from $\operatorname{Im} z>0$ to $\left\{z: \operatorname{Im} z>-a_{0}\right\} \backslash \mathscr{R}_{a_{0}}$ which is meromorphic in $\left\{z: \operatorname{Im} z>-a_{0}\right\}$. If $z_{0} \in \mathscr{R}_{a_{0}}$ then there is an $f \in \mathscr{D}$ such that $G(f, \cdot)$ has a pole at $z_{0}$.

The proof of Corollary 2.2 parallels the dilation analyticity proof in $[1,5]$. (See also [4]) Thus we do not give it here. Before proving Theorem 2.1, we make a few remarks:

1) We follow precedent and call the points of $\mathscr{R}_{a_{0}}$ resonances. Note that if we do not assume the electric field $F=1$, or the electric charge $e=1$, the Hamiltonian $H(\lambda)$ becomes

$$
H_{F}(\lambda)=-\frac{d^{2}}{d x^{2}}+V_{\lambda}(x)+e F x+e \lambda F
$$

and $\mathscr{R}_{a_{0}}$ becomes $\mathscr{R}_{\text {eF } a_{0}}$.

2) If $V$ is periodic, with period $\beta$ then

$$
U(\beta) H_{F}(\lambda) U(-\beta)=H_{F}(\lambda+\beta)=H_{F}(\lambda)+e \beta F
$$

and thus if $E_{0}$ is a resonance so is $E_{0}+n e \beta F$ for all integers $n$. This infinite sequence of poles with constant imaginary part is called the Stark ladder. Thus, if there is any resonance at all (which we have not shown), there must be a ladder. According to the lore, there is supposed to be a ladder for each conduction band, of which there are an infinite number. When $a_{0}=\infty$, then since resonances, in our sense, cannot accumulate at a finite point, the imaginary parts of an infinite number of ladders must tend to infinity. Thus, for high conduction bands, the ladder resonances must be far removed from the axis.

3) It should be possible to use our techniques to prove analyticity of the resonances in $F$ in some region away from $F=0$. We shall not attempt this here.

Proof of Theorem 2.1: Our technique of proof relies on a more or less explicit computation of the inverse of an operator of the form

$$
-\frac{d^{2}}{d x^{2}}+x+V_{\lambda}(x)+Q(z, \lambda, x)-z
$$

where for fixed $(z, \lambda), Q$ is a function of $x$ which vanishes at $\infty$. The solutions of the corresponding differential equation are WKB approximations to the original equation.

We then perturb this operator by subtracting $Q$. This procedure has much in common with Titchmarsh's treatment of the one-dimensional Stark problem [15] and the work of Rejto and Sinha [12] on Stark-like Hamiltonians.

The WKB approximation is based on the action integral, which in our case is

$$
\int \sqrt{x+V_{\lambda}(x)-z} d x \text {. }
$$

However, to prove analyticity in the variables $(z, \lambda)$, we find it easier to work with 
an asymptotic approximation to this, valid for large $|x|$. Accordingly, we define instead

$$
\begin{aligned}
S(z, \lambda ; x) & =\frac{2}{3} x^{3 / 2}-z x^{1 / 2}+\frac{1}{2} \int_{0}^{x} V_{\lambda}(t) t^{-1 / 2} d t, \quad x>0 \\
& =\frac{2}{3 i}(-x)^{3 / 2}-i z(-x)^{1 / 2}-\frac{i}{2} \int_{0}^{x} V_{\lambda}(t)(-t)^{-1 / 2} d t, \quad x<0 .
\end{aligned}
$$

For $|\operatorname{Im} \lambda|<a_{0}$ and $\operatorname{Im} z \neq 0$, define

$$
\phi^{+}(z, \lambda ; x)=e^{-S(z, \lambda ; x)} \quad x \geqq 1
$$

and

$$
\phi^{-}(z, \lambda ; x)=e^{\mp S(z, \lambda ; x)} \quad x \leqq-1
$$

if $\pm \operatorname{Im} z>0$. To be definite, consider $\operatorname{Im} z>0$; similar arguments work for $\operatorname{Im} z<0$. By direct computation, $\phi^{ \pm}$satisfy

$$
\left(-\frac{d^{2}}{d x^{2}}+x+V_{\lambda}(x)-z+Q(\lambda, z ; x)\right) \phi^{ \pm}=0
$$

where

$$
Q(\lambda, z ; x)=(4 x)^{-1}\left(V_{\lambda}(x)-z\right)^{2}-\frac{\partial^{2} S}{\partial x^{2}}(z, \lambda ; x)
$$

For small $x$ we modify the potential by defining:

$$
\begin{aligned}
Q_{\gamma}(z, \lambda ; x)= & \left\{\frac{1}{4 x}\left(z-V_{\lambda}(x)\right)^{2}-\frac{\partial^{2} S}{\partial x^{2}}(z, \lambda ; x)\right\}\left(1-\chi_{[-1,1]}(x)\right) \\
& -\chi_{[-1,1]}(x)\left(x+V_{\lambda}(x)-z\right)+\gamma \chi_{[-1 / 2,1 / 2]}(x)
\end{aligned}
$$

where $\chi_{A}$ is the indicator function of the set $A$. The reason for the parameter $\gamma$ will emerge later.

Note at this point that $Q_{\gamma}(z, \lambda, x)$ is bounded in $x$ for fixed $z, \lambda$ and $\gamma$. For Cauchy's formula

$$
V^{\prime}(z)=(2 \pi i)^{-1} \int_{|\zeta|=r} \zeta^{-2} V(z+\zeta) d \zeta
$$

and our assumptions yield the estimate

$$
\left|V^{\prime}(z)\right| \leqq c(a)(1+|z|)^{1 / 2-\varepsilon}
$$

for $z \in S_{a}, 0<a<a_{0}$, and this yields an estimate

$$
\left|Q_{\gamma}(z, \lambda ; x)\right| \leqq c(\gamma, z, \lambda)(1+|x|)^{-\varepsilon}
$$

where $c(\gamma, z, \lambda)$ is bounded uniformly on compact subsets of $\mathbb{C} \times \Gamma$ with $\Gamma=\{z: \operatorname{Im} z>0\} \times S_{a_{0}}$.

We now define $\phi_{\gamma}^{ \pm}(z, \lambda ; x)$ respectively to be the solution of

$$
\left(-\frac{d^{2}}{d x^{2}}+x+V_{\lambda}(x)-z+Q_{\gamma}(z, \lambda ; x)\right) \phi_{\gamma}^{ \pm}(z, \lambda ; x)=0
$$


for all $x$ which is equal to $\phi^{ \pm}(z, \lambda ; x)$ for $\pm x \geqq 1$. Let

$$
G_{\gamma}(z, \lambda ; x, y)=\phi_{\gamma}^{+}\left(z, \lambda ; x_{>}\right) \phi_{\gamma}^{-}\left(z, \lambda ; x_{<}\right)
$$

where $x_{>}=\max (x, y)$ and $x_{<}=\min (x, y)$. It is shown in the Appendix that the operator

$$
A_{\gamma}(z, \lambda) f(x)=\int_{-\infty}^{+\infty} G_{\gamma}(z, \lambda ; x, y) f(y) d y
$$

is bounded for $\gamma \in \mathbb{C}$, and $(z, \lambda) \in \Gamma$, with norm uniformly bounded on compact subsets of $\Gamma$, for $\gamma$ fixed. From analyticity of $\phi_{\gamma}^{ \pm}(z, \lambda ; x)$ and the uniform bound, it follows that $A_{\gamma}(z, \lambda)$ is a bounded analytic family of operators in $\Gamma$.

Let $W_{\gamma}(z, \lambda)$ be the Wronskian of $\phi_{\gamma}^{+}(z, \lambda ; x)$ and $\phi_{\gamma}^{-}(z, \lambda ; x)$. It is easy to see that for any $(z, \lambda), \gamma$ can be chosen so that $W_{\gamma}(z, \lambda) \neq 0$. Note also that $W_{\gamma}$ is analytic in the variables $(z, \lambda)$ for $(z, \lambda) \in \Gamma$. Fix $(z, \lambda) \in \Gamma$ and choose $\gamma$ so that $W_{\gamma}(z, \lambda) \neq 0$. Define the operators

$$
\tilde{A}_{\gamma}(z, \lambda) \equiv\left(W_{\gamma}(z, \lambda)\right)^{-1} A_{\gamma}(z, \lambda)
$$

and

$$
B_{\gamma}(z, \lambda)=-\frac{d^{2}}{d x^{2}}+x+V_{\lambda}(x)+Q_{\gamma}(z, \lambda ; x)-z
$$

with domain $\mathscr{D}\left(B_{\gamma}(z, \lambda)\right)=C_{0}^{\infty}(\mathbb{R})$. Integration by parts gives

$$
\begin{array}{r}
\tilde{A}_{\gamma}(z, \lambda) B_{\gamma}(z, \lambda) f=f \\
\tilde{A}_{\gamma}(z, \lambda)^{*} B_{\gamma}(z, \lambda)^{*} f=f
\end{array}
$$

for $f \in C_{0}^{\infty}(\mathbb{R})$.

By (2.7), Ran $\tilde{A}_{\gamma}^{*}$ is dense so that Ker $\tilde{A}_{\gamma}=\{0\}$. Equation (2.6) then implies that $B_{\gamma}(z, \lambda)$ is closable; for if $f_{n} \in C_{0}^{\infty}, f_{n} \rightarrow 0$, and $B_{\gamma}(z, \lambda) f_{n} \rightarrow g$, then $\tilde{A}_{\gamma}(z, \lambda) g=0$, and so $g=0$. Hence (2.6) extends to

$$
\tilde{A}_{\gamma}(z, \lambda) \bar{B}_{\gamma}(z, \lambda) f=f, \quad f \in \mathscr{D}\left(\bar{B}_{\gamma}(z, \lambda)\right)
$$

where $\bar{B}_{\gamma}(z, \lambda)$ is the closure of $B_{\gamma}(z, \lambda)$. This implies that $\operatorname{Ran} \bar{B}_{\gamma}(z, \lambda)$ is closed. Moreover, $\operatorname{Ker} B_{\gamma}(z, \lambda)^{*}=\{0\}$, for $B_{\gamma}(z, \lambda)^{*} f=0$ implies (by elliptic regularity!) that $\bar{f}$ is an $L_{2}$ solution of

$$
\left[-\frac{d^{2}}{d x^{2}}+x+V_{\lambda}(x)+Q_{\gamma}(z, \lambda ; x)\right] \bar{f}=0
$$

which is shown in the Appendix to imply that $\bar{f}$ vanishes identically. Hence, $\bar{B}_{\gamma}(z, \lambda)$ is surjective, and has the bounded inverse

$$
\bar{B}_{\gamma}(z, \lambda)^{-1}=\tilde{A}_{\gamma}(z, \lambda) .
$$

It now follows that the restriction of

$$
H(\lambda)=B_{\gamma}(z, \lambda)-Q_{\gamma}(z, \lambda ; x)+\lambda
$$

to $C_{0}^{\infty}(\mathbb{R})$ is closable, since $Q_{\gamma}$ is bounded. The fact that $U(a) H(\lambda) U(-a)=H(\lambda+a)$ follows from the definition of $H(\lambda)$. This proves (i). 
Let

$$
K_{\gamma}(z, \lambda)=Q_{\gamma}(z, \lambda) \tilde{A}_{\gamma}(z, \lambda)
$$

where $Q_{\gamma}(z, \lambda)$ is multiplication by $Q_{\gamma}(z, \lambda ; x)$. We claim that $K_{\gamma}(z, \lambda)$ is compact. By a limiting argument (and the bound (2.3)) we need only show that

$$
g A_{\gamma}(z, \lambda)
$$

is compact for $g \in C_{0}^{\infty}$.

But this operator is Hilbert-Schmidt. In fact, if $g$ is supported in $[-a, a]$, the Hilbert-Schmidt norm of the $y>x$ half of this operator is

$$
\begin{aligned}
& \int_{-a}^{a} \int_{x}^{\infty}|g(x)|^{2}\left|\phi_{-}(x)\right|^{2}\left|\phi_{+}(y)\right|^{2} d y d x \\
& \leqq\left(\int_{-a}^{a}|g(x)|^{2}\left|\phi_{-}(x)\right|^{2} d x\right)\left(\int_{a}^{\infty}\left|\phi_{+}(y)\right|^{2} d y\right)<\infty
\end{aligned}
$$

and similarly for the other half.

We now prove (iii) of the theorem. Let

$$
\hat{H}(\lambda)=H(\lambda)-\lambda
$$

and note that for $b$ real

$$
U(b) \hat{H}(\lambda) U(-b)=\hat{H}(\lambda+b)+b .
$$

We claim that

$$
\sigma_{\text {ess }}(\hat{H}(\lambda)) \subseteq \mathbb{R}
$$

for all $\lambda \in S_{a_{0}}$. Define $\Sigma$ to be the set of all $\lambda \in S_{a_{0}}$ such that $(\hat{H}(\lambda)-z)^{-1}$ is meromorphic in $\operatorname{Im} z>0$, with finite rank principal parts at every pole. We shall prove that $\Sigma=S_{a_{0}}$. For choose $\gamma$ such that $W_{\gamma}(i, 0) \neq 0$, so that $\bar{B}_{\gamma}(i, 0)$ is invertible. We then have

$$
H-i=\bar{B}_{\gamma}(i, 0)-Q_{\gamma}(i, 0)=\left[1-K_{\gamma}(i, 0)\right] \bar{B}_{\gamma}(i, 0) .
$$

Since $H$ is selfadjoint, $(H-i)$ is invertible, and so $1-K_{\gamma}(i, 0)$ is also invertible. By the analytic Fredholm theorem ([11, Theorem IV. 14] or $[13]) 1-K_{\gamma}(i, \lambda)$ is invertible, and $W_{\nu}(i, \lambda)$ does not vanish, for every $\lambda \in S_{a_{0}} \backslash T$ where $T$ is discrete. For $\lambda$ in this set, $\left[I-K_{\gamma}(z, \lambda)\right]^{-1}$ is meromorphic on $\operatorname{Im} z>0$, with finite rank principal parts at its poles; so the same is true for

$$
(\hat{H}(\lambda)-z)^{-1}=W_{\gamma}^{-1}(z, \lambda) A_{\gamma}(z, \lambda)\left(I-K_{\gamma}(z, \lambda)\right)^{-1} .
$$

Hence, $S_{a_{0}} \backslash T \subseteq \Sigma$. But by (2.11), $\Sigma$ is closed under real translates, so the points of $T$ are in $\Sigma$, too. This shows that $\sigma_{\text {ess }}(\hat{H}(\lambda))$ does not intersect $\operatorname{Im} z>0$. By a similar argument, it does not intersect $\operatorname{Im} z<0$, so (2.12) holds. This proves (iii) of the theorem.

We now concentrate on part (v) of the theorem. We show that given $z_{0}$ and a point $\lambda_{0} \in E_{z_{0}},\left(z_{0}-H(\lambda)\right)^{-1}$ is analytic for $\lambda$ in a neighborhood of $\lambda_{0}$. This and a similar argument for $D_{z}$ will prove $(\mathrm{v})$. Thus choose $\gamma$ so that $W_{\gamma}\left(z_{0}-\lambda_{0}, \lambda_{0}\right) \neq 0$. 
Because $W_{\gamma}\left(z_{0}-\lambda, \lambda\right)$ is analytic in $\lambda$ in a neighborhood of $\lambda_{0}$, we have $\left|W_{\gamma}\left(z_{0}-\lambda, \lambda\right)\right|>0$ for $\left|\lambda-\lambda_{0}\right|<\delta$. Note that since $H\left(\lambda_{0}\right)-z_{0}=$ $\hat{H}\left(\lambda_{0}\right)-\left(z_{0}-\lambda_{0}\right)$ is invertible and

$$
\hat{H}\left(\lambda_{0}\right)-\left(z_{0}-\lambda_{0}\right)=\left(1-K_{\gamma}\left(z_{0}-\lambda_{0}, \lambda_{0}\right)\right) \bar{B}_{\gamma}\left(z_{0}-\lambda_{0}, \lambda_{0}\right)
$$

we have $1-K_{\gamma}\left(z_{0}-\lambda_{0}, \lambda_{0}\right)$ is invertible and thus by continuity $1-K_{\gamma}\left(z_{0}-\lambda, \lambda\right)$ is invertible in a neighborhood of $\lambda_{0}$. Hence

$$
\left(H(\lambda)-z_{0}\right)^{-1}=\tilde{A}_{\gamma}\left(z_{0}-\lambda, \lambda\right)\left(1-K_{\gamma}\left(z_{0}-\lambda, \lambda\right)\right)^{-1}
$$

in a neighborhood $\left|\lambda-\lambda_{0}\right|<\delta^{\prime}$ of $\lambda_{0}$ where the right hand side is analytic in $\lambda$. This proves (v).

To prove (iv), first note that $\sigma(H(\lambda))=\sigma(H(\lambda+\mu))$ if $\mu$ is real (see (i)) and since the isolated eigenvalues of $H(\lambda)$ (away from $\mathbb{R}+i \operatorname{Im} \lambda$ ) are branches of functions of $\lambda$ with at most algebraic singularities, they are independent of $\lambda$ (as long as the line $\mathbb{R}+i \operatorname{Im} \lambda$ does not intersect these eigenvalues). Since $H(0)$ is self-adjoint, if $a_{0}>\operatorname{Im} \lambda>0, H(\lambda)$ has no eigenvalues in $\{z: \operatorname{Im} z>\operatorname{Im} \lambda$ or $\operatorname{Im} z<0\}$, since if there were their constancy would imply they remained eigenvalues for $H(0)$. This proves (iv).

To prove (ii), suppose $\lambda_{0} \in S_{a}$ is given with $\operatorname{Im} \lambda_{0}<0$ and suppose $f, g$ rare entire vectors for the translation group. Denote by $f_{\lambda}$ the analytic continuation of $U(\lambda) f$ from $\lambda \in \mathbb{R}$ to $\mathbb{C}$. Then for $\operatorname{Im} z>0$

$$
\left(f,(z-H)^{-1} g\right)=\left(f_{\bar{\lambda}},(z-H(\lambda))^{-1} g_{\lambda}\right) \quad \lambda \in \mathbb{R}
$$

and since the right hand side is analytic in $\lambda$ for $\lambda \in S_{a_{0}} \cap\{\lambda: \operatorname{Im} \lambda<\operatorname{Im} z\}$ we have

$$
\left(f,(z-H)^{-1} g\right)=\left(f_{\bar{\lambda}_{0}},\left(z-H\left(\lambda_{0}\right)\right)^{-1} g_{\lambda_{0}}\right)
$$

Similarly

$$
\left(g,(\bar{z}-H)^{-1} f\right)=\left(g_{\lambda_{0}},\left(\bar{z}-H\left(\bar{\lambda}_{0}\right)\right)^{-1} f_{\bar{\lambda}_{0}}\right) .
$$

Taking the complex conjugate of (2.14) gives

$$
\left(g,(\bar{z}-H)^{-1} f\right)=\left(g_{\lambda_{0}},\left(\bar{z}-H\left(\lambda_{0}\right)^{*}\right)^{-1} f_{\lambda_{0}}\right) .
$$

Comparing this to (2.15), we have equality of the matrix elements of $\left(\bar{z}-H\left(\bar{\lambda}_{0}\right)\right)^{-1}$ and $\left(\bar{z}-H\left(\lambda_{0}\right)^{*}\right)^{-1}$ for a dense set of vectors. Hence $H\left(\lambda_{0}\right)^{*}=H\left(\bar{\lambda}_{0}\right)$.

This completes the proof of Theorem 2.1.

\section{A Random Potential}

Let the probability space $(P, \Omega, \mathscr{F})$ correspond to a Poisson ensemble of points in $\mathbb{R}$, with mean density $\alpha$. For each $\omega$, let $X_{j}(\omega), j=0, \pm 1, \pm 2, \ldots$ be the points of the Poisson set, numbered so that

$$
\ldots<X_{-1}<0<X_{0}<X_{1}<\ldots
$$

This defines $X_{j}(\omega)$ a.s. The number $N[S]$ of points $X_{j}$ in the Borel set $S$ is then Poisson, with mean $\alpha|S|$, where $|S|$ is the Lebesgue measure of $S$. The variables $N(S)$ for disjoint sets $S$ are independent. 
Let $u(x)$ be a real-valued function on $\mathbb{R}$, representing the potential of a certain type of particle, centered at the origin. If an infinite number of such particles are stationed at the points of a Poisson set, their total potential is

$$
V(x, \omega)=\sum_{j=-\infty}^{+\infty} u\left(x-X_{j}(\omega)\right) .
$$

The goal of this section is to prove Theorem 3.6 which verifies that $V(x, \omega)$ satisfies the conditions of Theorem 2.1 almost surely for a wide class of $u$.

In preparation, we shall prove a number of properties of the process $V(x, \omega)$, from which the result follows easily. Unless we state the contrary, $u(x)$ is assumed below to be complex valued.

\subsection{Proposition Let $u(x)$ be bounded and integrable on $\mathbb{R}$. Then}

(a) $V(x)$ is a well defined, strictly stationary process, with mean

$$
E V(x)=\alpha \int_{-\infty}^{+\infty} u(y) d y
$$

and variance

$$
E|V(x)-E V(x)|^{2}=\alpha \int_{-\infty}^{+\infty}|u(y)|^{2} d y
$$

(b) For a.e. $\omega, V(x, \omega)$ is a finite-valued measurable function of $x$, and

$$
\lim _{T \rightarrow \infty} \frac{1}{T} \int_{0}^{T} V(x, \omega) d x=\alpha \int_{-\infty}^{+\infty} u(y) d y \quad \text { a.s. }
$$

Proof: (a) By linearity, we may assume $u(x) \geqq 0$. If $u_{n}(x) \uparrow u(x)$, then the corresponding sequence $V_{n} \uparrow V$ for all $x$ and $\omega$, so it suffices to prove the result for simple functions, and hence for indicators. If $u=1_{S}$ for some Borel set $S$, then $V(x)=$ $N([-S+x])$, the number of points $X_{j}$ in $-S+x$, which is Poisson with mean

$$
E V(x)=\alpha|S-x|=\alpha|S|=\alpha \int_{-\infty}^{+\infty} u(y) d y .
$$

This shows that $V(x, \omega)$ is a.s. finite for each $x$.

The formula for variance follows if we establish that for two such functions $u$ and $u_{1}$,

$$
\operatorname{cov}\left(V(x), V_{1}(y)\right)=\alpha \int_{-\infty}^{+\infty} \bar{u}(x-y+s) u_{1}(s) d s .
$$

It suffices again to consider indicators, and to take $x=y=0$. Put $u=1_{-S}, u_{1}=1_{-J}$, and let $S J=S \cap J$. Writing

$$
N[S] N[J]=(N[S \backslash J]+N[S J])(N[J \backslash S]+N(S J))
$$

yields

$$
\operatorname{cov}(N[S] N[J])=E N[S] N[J]-(E N[S])(E N[J])
$$




$$
\begin{aligned}
& =\alpha^{2}\{|S \backslash J||J \backslash S|+|S J|(|J \backslash S|+|S \backslash J|)\}+\alpha^{2}|S J|^{2}+\alpha|S J|-\alpha^{2}|S||J| \\
& =\alpha|J S|=\alpha \int_{-\infty}^{+\infty} u(S) u_{1}(s) d s .
\end{aligned}
$$

(b) Stationarity of $V(x)$ for $u=1_{s}$ is simply stationarity of the Poisson ensemble. Again, if $u$ has compact support with diameter $d$, then $V(x)$ and $V(y)$ are independent if $|x-y|>2 d$, since they depend on $N(S)$ in disjoint regions. The process $V(x)$ is therefore ergodic (even mixing), and (3.4) follows from the Birkhoff ergodic theorem. The general case follows by an $L_{1}$ approximation. For by (3.2), if $u_{n} \rightarrow u$ in $L_{1}(\mathbb{R})$, then

$$
\sup _{x \in \mathbb{R}} E\left|V_{n}(x)-V(x)\right| \rightarrow 0 .
$$

The limiting process $V(x)$ is again stationary, and both sides of (3.4) are stable under this approximation.

Remarks. The formulas for the mean and variance of $V(x)$ are essentially Campbell's theorem for the shot effect in vacuum tubes [8]. The only difference is that there one is dealing with a Poisson ensemble on $[0, \infty)$ rather than on $\mathbb{R}$ as here

3.2 Lemma Let $N_{1}, N_{2}, \ldots$ be independent Poisson variables with mean $\alpha$, and $c_{1}, c_{2}, \ldots$ a sequence of non-negative numbers with $0<\sum_{k=1}^{\infty} c_{k}=C<\infty$. Let $Y=\sum c_{k} N_{k}$. Then there exist positive constants $\gamma$ and $\beta$ dependent only on $C, m=$ $\sup \left\{c_{k}: k \geqq 1\right\}$ and $\alpha$ such that for all $\lambda>0$

$$
P\{Y \geqq \lambda\} \leqq \lambda^{-\gamma \lambda} e^{\beta \lambda}
$$

Proof. The moment generating function of $Y$ is

$$
E e^{t Y}=\prod_{k=1}^{\infty} E e^{t c_{k} N_{k}}=\prod_{k=1}^{\infty} \exp \alpha\left\{e^{c_{k} t}-1\right\}=\exp \left\{\alpha \sum_{k=1}^{\infty}\left(e^{c_{k} t}-1\right)\right\}
$$

Using $e^{x}-1 \leqq x e^{x}$ for $x \geqq 0$ we have

$$
E e^{t Y} \leqq \exp \left\{\alpha \sum_{k=1}^{\infty} c_{k} t e^{c_{k} t}\right\} \leqq \exp \left\{\alpha C t e^{m t}\right\}
$$

where $m=\max \left\{c_{1}, c_{2}, \ldots\right\}$. Hence, by Chebyshev's inequality

$$
P\{Y \geqq \lambda\}=P\left\{e^{t Y} \geqq e^{t \lambda}\right\} \leqq e^{-t \lambda} E e^{t Y} \leqq \exp (f(t, \lambda))
$$

where

$$
f(t, \lambda)=\alpha C t e^{m t}-\lambda t .
$$

If $\lambda \geqq \alpha C, f(t, \lambda)$ is minimized by choosing $t$ to satisfy $\alpha C e^{m t}(1+m t)=\lambda$, in which case $f(t, \lambda)=-\lambda t\left(\frac{m t}{1+m t}\right)$. We have $\alpha C e^{m t}(1+m t) \leqq \alpha C e^{2 m t}$ so that $2 m t \geqq$ 
$\log (\lambda / \alpha C)$. If we take $\lambda \geqq e \alpha C, f(t, \lambda) \leqq-\left(\frac{1}{6 m}\right) \lambda \log (\lambda / \alpha C) \leqq-\frac{1}{6 m} \lambda \log \lambda+\frac{1}{6 m} \lambda$ $\log e \alpha C$. Thus the lemma holds with $\gamma=(6 m)^{-1}$ and $\beta=(6 m)^{-1} \log e \alpha C$.

This lemma yields an estimate for the tail of the distribution of $V(x)$. For if we write

$$
V(x)=\sum_{n=-\infty}^{\infty} \sum_{n \leqq X_{j}<n+1} u\left(x-X_{j}\right)
$$

then if $x \in[0,1]$ we obtain the estimate

$$
|V(x)| \leqq \sum_{n=-\infty}^{\infty} c_{n} N[n, n+1)
$$

with $c_{n}=\sup \{|u(x)|:|x+n| \leqq 1\}$. Thus we have:

3.3. Proposition (a) If

$$
\sum_{n=-\infty}^{\infty} \sup _{|x+n| \leqq 1}|u(x)|<\infty
$$

then there are positive constants $\gamma$ and $\beta$ such that for $\lambda>0$

$$
P\left\{\sup _{0 \leqq x \leqq 1}|V(x)| \geqq \lambda\right\} \leqq \lambda^{-\gamma \lambda} e^{\beta \lambda}
$$

and the series (3.6) converges uniformly for $0 \leqq x \leqq 1$ almost surely.

(b) If $V(x)=V(x, a)$ depends on a parameter a lying in a set $S$, and

$$
\sum_{n=-\infty}^{\infty} \sup \{|u(x)|:|x+n| \leqq 1, a \in S\}<\infty
$$

then (3.9) holds for $\sup \{|V(x)|: 0 \leqq x \leqq 1, a \in S\}$ and there is uniform convergence on $[0,1] \times S$.

Proof. Part (a) is immediate from Lemma 3.2 and (3.7). Part (b) follows by applying (a) to the function $u_{0}(x)=\sup \{|u(x, a)|: a \in S\}$.

Remark. From the uniform convergence, it follows that $V(x)$ is a.s. bounded on every finite interval whenever (3.8) holds. If $u$ is continuous, then $V$ will be continuous a.s., while if $u \in C^{n}$, and all $n$ derivatives satisfy (3.8), then the differentiated series also converge uniformly, so that $V$ is in $C^{n}$ a.s.

3.4. Proposition (a) Let (3.8) hold, and let $\phi(x)$ be a positive even function, increasing for $x>0$, with $\lim _{x \rightarrow \infty} \phi(x)=\infty$. If

$$
\int_{0}^{\infty} e^{-(1 / 2) \gamma \phi(x) \log \phi(x)} d x<\infty
$$

where $\gamma$ is the constant of (3.9), then

$$
\lim _{|x| \rightarrow \infty} \sup \frac{|V(x, \omega)|}{\phi(x)} \leqq 1 \text { a.s. }
$$


(b) If (3.10) holds, $V(x)$ can be replaced in (3.11) by sup $\left\{\left|V_{a}(x, \omega)\right|: a \in S\right\}$.

Proof. Let $a_{n}=\phi(n)$. By Proposition 3.3,

$$
\begin{aligned}
P\{|V(x)| & \geqq \phi(x) \text { for some } x, n-1 \leqq|x|<n\} \\
& \leqq P\left\{\sup _{n-1 \leqq|x|<n}|V(x)| \geqq a_{n}\right\} \leqq a_{n}^{-\gamma a_{n}} e^{\beta a_{n}} .
\end{aligned}
$$

By Borel-Cantelli, if

$$
\sum a_{n}^{-\gamma a_{n}} e^{\beta a_{n}}<\infty
$$

then one has $|V(x)| \geqq \phi(x)$ on infinitely many intervals $n-1 \leqq|x|<n$ with probability zero, which is simply (3.11) restated. However, if $n$ is large enough that $a_{n} \geqq e^{2 \beta / \gamma}$, then

$$
a_{n}^{-\gamma a_{n}} e^{\beta a_{n}} \leqq \exp \left\{-\frac{\gamma}{2} \phi(n) \log \phi(n)\right\}
$$

so that (3.12) holds by the integral test.

3.5. Corollary. If (3.8) holds, then, as $|x| \rightarrow \infty$,

$$
V(x, \omega)=0(\log |x|) \quad \text { a.s. }
$$

If (3.10) holds, this is true uniformly for $a \in S$.

Proof. For large $x, \frac{1}{2} \gamma \log \log x \geqq \delta>1$, so that

$$
\exp \left\{-\frac{1}{2} \gamma \log x(\log \log x)\right\} \leqq x^{-\delta}
$$

which is integrable at infinity.

Remark. Although (3.13) looks like, and is, a growth estimate for $V(x)$, it is worth pointing out that by the ergodic theorem

$$
\lim _{T \rightarrow \infty} \frac{1}{T} \int_{0}^{T} V(x) d x=E V(x)=\alpha \int_{-\infty}^{+\infty} u(x) d x
$$

a.s., so that $V(x)$ by no means really grows at infinity. What happens is that $V(x)$ may have large bumps near points where many $X_{j}$ 's are clustered. Equation (3.4) says that one must go a long way out to find a big bump.

3.6. Theorem. Let $u(z)$ be analytic in the strip $|\operatorname{Im} z|<a_{0}, 0<a_{0} \leqq \infty$, and real for real $z$. Assume that

$$
\int_{-\infty}^{+\infty}|u(x+i a)| d x<\infty
$$

for $-a_{0}<a<a_{0}$. Then the potential

$$
V(z, \omega)=\sum_{j} u\left(z-X_{j}(\omega)\right)
$$

satisfies the conditions of Theorem 2.1 for a.e. $\omega$. 
Proof. Fix two arbitrary numbers $a_{1}$ and $b$ satisfying $0<a_{1}<b<a_{0}$. By the three lines theorem,

$$
\int_{-\infty}^{+\infty}|u(x+i a)| d x
$$

is bounded uniformly for $-b \leqq a \leqq b$. Hence

$$
\int_{-b}^{b} \int_{-\infty}^{+\infty}|u(x+i a)| d x d a=\sum_{n=-\infty}^{+\infty} \int_{0}^{1} d x \int_{-b}^{b} d a|u(x+n+i a)|
$$

is finite, so there must exist an $x_{0}$ with

$$
\sum_{n=-\infty}^{+\infty} \int_{-b}^{b}\left|u\left(x_{0}+n+i a\right)\right| d a<\infty
$$

By relabelling, we can take $x_{0}=0$, so that

$$
\sum_{n=-\infty}^{+\infty} \int_{-b}^{b}|u(n+i a)| d a<\infty
$$

Let $\Gamma$ be the boundary of the rectangle with vertices $\pm 2 \pm i b$. Then for $|x|<2$ and $|a|<b$, we have

$$
u(x+n+i a)=(2 \pi i)^{-1} \int_{\Gamma}(\zeta-x-i a)^{-1} u(\zeta+n) d \zeta
$$

where the integral is taken in the positive sense. If we restrict to $|x| \leqq 1$ and $|a| \leqq a_{1}$, we obtain the estimate

$$
|u(x+n+i a)| \leqq c_{n}
$$

where

$$
\begin{aligned}
c_{n}= & c \int_{-2}^{2}(|u(x+n+i b)|+|u(x+n-i b)|) d x \\
& +c \int_{-b}^{b}(|u(2+n+i a)|+|u(-2+n+i a)|) d a
\end{aligned}
$$

and $c$ depends only on $a_{1}$ and $b$. By (3.14) and (3.15),

$$
\sum_{n=-\infty}^{+\infty} c_{n}<\infty
$$

and so

$$
\sum_{n=-\infty}^{+\infty} \sup \left\{|u(x+n+i a)|:-1 \leqq x \leqq 1,-a_{1} \leqq a \leqq a_{1}\right\}<\infty .
$$

Mutatis mutandis, this is (3.10), with $S=\left[-a_{1}, a_{1}\right]$. We therefore obtain uniform convergence on compact subsets of the strip by Proposition 3.3 and a bound

$$
|V(x+i a)| \leqq C \log (|x|+2)
$$

for $-a_{1} \leqq a \leqq a_{1}$, almost surely. Since $V(x)$ is obviously real for real $x$, this completes the proof. 
Remarks. (1) For $F \neq 0$, it is proved in [18] that

$$
H_{F}(\omega)=-\frac{d^{2}}{d x^{2}}+F x+V(x, \omega)
$$

is almost surely spectrally absolutely continuous. This means physically that particles will propagate.

(2) For some random potentials, it is known rigorously that for zero field $H_{0}(\omega)$ has pure point spectrum a.s. and the same result has been claimed for our potential with $u(x)$ replaced by a delta function $[9,10]$. Although our $V(x, \omega)$ does not appear to satisfy the hypotheses of $[9,10]$, it is a reasonable conjecture that $H_{0}(\omega)$ also has pure point spectrum a.s. If this is true, then intuition might lead one to expect for small field $F$ a large number of resonances near any fixed interval of the axis. A result in this direction would be quite interesting.

(3) For $F=0$ it should not be difficult to show that the spectrum of $H_{0}(\omega)$ is $[0, \infty)$ if $u(x) \geqq 0$, and is $(-\infty, \infty)$ if $u(x)<0$ on a set of positive measure.

\section{Appendix}

Proof of Boundedness of $A_{\gamma}(z, \lambda)$ and $\operatorname{Ker} B_{\gamma}^{*}(z, \lambda)=\{0\}$

Proposition A.1. Suppose $\gamma$ is fixed and $\Lambda \subseteq \Gamma=\{z: \operatorname{Im} z>0\} \times S_{a_{0}}$ is compact. Then

$$
\sup _{(z, \lambda) \in \Lambda}\left\|A_{\gamma}(z, \lambda)\right\|<\infty .
$$

The proof of this proposition rests on explicit estimates on the solutions $\phi_{\gamma}^{ \pm}(z, \lambda ; x)$ of the differential equation

$$
\left(-\frac{d^{2}}{d x^{2}}+x+V_{\lambda}(x)+Q_{\gamma}(z, \lambda ; x)\right) f=0 .
$$

These estimates will also lead to

Proposition A.2. Suppose $W_{\gamma}(z, \lambda) \neq 0$. Then there are no $L^{2}$ solutions of the differential equation (A.1).

Since we know $\phi_{\gamma}^{ \pm}$explicitly only for $\pm x>1$ respectively we introduce the solutions $\psi_{\gamma}^{ \pm}$defined by

$$
\psi_{\gamma}^{+}(z, \lambda ; x)=\phi_{\gamma}^{+}(z, \lambda ; x) \int_{1}^{x} \frac{1}{\left[\phi_{\gamma}^{+}(z, \lambda ; t)\right]^{2}} d t \quad x \geqq 1
$$

and

$$
\psi_{\gamma}^{-}(z, \lambda ; x)=\phi_{\gamma}^{-}(z, \lambda ; x) \int_{-1}^{x} \frac{1}{\left[\phi_{\gamma}^{-}(z, \lambda ; t)\right]^{2}} d t \quad x \leqq-1 .
$$
and $\phi_{\gamma}^{-}$.

Note that the Wronskian of $\psi_{\gamma}^{+}$and $\phi_{\gamma}^{+}$is 1 as is the Wronskian of $\psi_{\gamma}^{-}$

We are assuming throughout that assumptions a), b) and c) of Sect. 2 concerning the potential $V$ are in force. 
Lemma A.3. There exist constants $c_{1}, c_{2}>0$ so that for all $(z, \lambda) \in \Lambda$ and $x \geqq 1$

$$
\begin{aligned}
\left|\psi_{\gamma}^{+}(z, \lambda ; x)\right| & \leqq c_{1} x^{-1 / 2} \exp \left\{\frac{2}{3} x^{3 / 2}-\operatorname{Re} z x^{1 / 2}+\frac{1}{2} \operatorname{Re} \int_{0}^{x} V_{\lambda}(t) \frac{d t}{\sqrt{t}}\right\} \\
& \leqq c_{2}\left|\psi_{\gamma}^{+}(z, \lambda ; x)\right|
\end{aligned}
$$

(ii) $\left|\psi_{\gamma}^{-}(z, \lambda ;-x)\right| \leqq c_{1} x^{-1 / 2} \exp \left\{(\operatorname{Im} z) x^{1 / 2}\right\} \leqq c_{2}\left|\psi_{\gamma}^{-}(z, \lambda ;-x)\right|$.

Proof of Lemma A.3. We have

$$
\int_{1}^{x}\left|\left(\phi_{\gamma}^{+} z, \lambda ; t\right)\right|^{-2} d t=\int_{1}^{x} \exp \left\{\frac{4}{3} t^{3 / 2}-2 \operatorname{Re} z t^{1 / 2}+\operatorname{Re} \int_{0}^{t} \frac{V_{\lambda}(s)}{\sqrt{s}} d s\right\} d t .
$$

We introduce the variable $y=t^{3 / 2}$ and write this as

$$
\int_{1}^{x^{3 / 2}} M(y) d y
$$

where

$$
M(y)=\frac{2}{3} y^{-1 / 3} \exp \left\{\frac{4}{3} y-2 \operatorname{Re} z y^{1 / 3}+\operatorname{Re} \int_{0}^{y^{2 / 3}} \frac{V_{\lambda}(s) d s}{\sqrt{s}}\right\} .
$$

We integrate by parts (integrate $\exp \left(\frac{4}{3} y\right)$, differentiate the rest) and find

$$
\begin{gathered}
\int_{1}^{x^{3 / 2}} M(y) d y=F(x)+\int_{1}^{x^{3 / 2}} M(y) g(y) d y \\
F(x)=\frac{1}{2} x^{-1 / 2} \exp \left\{\frac{4}{3} x^{3 / 2}-2 \operatorname{Re} z x^{1 / 2}+\operatorname{Re} \int_{0}^{x} \frac{V_{\lambda}(t) d t}{\sqrt{t}}\right\}+\text { const }
\end{gathered}
$$

and

$$
g(y)=\frac{1}{2} \operatorname{Re} z y^{-2 / 3}+\frac{1}{4} y^{-1}-\frac{1}{2} \operatorname{Re} V\left(y^{2 / 3}\right) y^{-2 / 3} .
$$

If $y_{0}$ is large enough so that $|g(y)| \leqq \frac{1}{2}$ if $y \geqq y_{0}$ we have (for $x^{3 / 2} \geqq y_{0}$ )

$$
\begin{aligned}
\int_{1}^{x^{3 / 2}} M(y) d y & \leqq F(x)+\text { const }+\frac{1}{2} \int_{y_{0}}^{x^{3 / 2}} M(y) d y \\
& \leqq F(x)+\text { const }+\frac{1}{2} \int_{1}^{x^{3 / 2}} M(y) d y
\end{aligned}
$$

so that $\int_{1}^{x^{3 / 2}} M(y) d y \leqq 2 F(x)+$ const.

Similarly

$$
\int_{1}^{x^{3 / 2}} M(y) d y \geqq F(x)-\text { const }-\frac{1}{2} \int_{y_{0}}^{x^{3 / 2}} M(y) d y
$$

so that $\int_{1}^{x^{3 / 2}} M(y) d y \geqq \frac{2}{3} F(x)-$ const. This proves (i) when we note all constants can be chosen independent of $(z, \lambda) \in \Lambda$. 
Now consider

$$
\alpha(x)=\int_{-1}^{-x}\left[\phi_{\gamma}^{-}(z, \lambda ; t)\right]^{-2} d t \quad x \geqq 1
$$

we have

$$
\begin{aligned}
\alpha(x) & =-\int_{1}^{x} \exp \left\{-i \frac{4}{3} t^{3 / 2}-2 i z t^{1 / 2}+i \int_{0}^{t} V_{\lambda}(-s) \frac{d s}{\sqrt{s}}\right\} d t \\
& =-\frac{2}{3} \int_{1}^{x^{3 / 2}} N(y) y^{-1 / 3} d y
\end{aligned}
$$

where

$$
N(y)=\exp \left(-\frac{4 i}{3} y-2 i z y^{1 / 3}+i \int_{0}^{y^{2 / 3}} V_{\lambda}(-s) \frac{d s}{\sqrt{s}}\right) .
$$

Integrating by parts we have

$$
\alpha(x)=G(x)+\frac{i}{2} \int_{1}^{x^{3 / 2}} N(y) h(y) y^{-1 / 3} d y
$$

where

$$
G(x)=(2 i)^{-1} x^{-1 / 2} \exp \left(-\frac{i 4}{3} x^{3 / 2}-2 i z x^{1 / 2}+i \int_{0}^{x} V_{\lambda}(-s) \frac{d s}{\sqrt{s}}\right)+\text { const }
$$

and

$$
h(y)=-\frac{2 i z}{3} y^{-2 / 3}+\frac{2 i}{3} V_{\lambda}\left(-y^{2 / 3}\right) y^{-2 / 3}-\frac{1}{3} y^{-1} .
$$

Integrating by parts again we have

$$
\int_{1}^{x^{3 / 2}} N(y) h(y) y^{-1 / 3} d y=G_{1}(x)+\text { const. } \int_{1}^{x^{3 / 2}} N(y) y^{-1 / 3}\left(h(y)^{2}+h^{\prime}(y)\right) d y
$$

where

$$
G_{1}(x)=\text { const } x^{-1 / 2} h\left(x^{3 / 2}\right) N\left(x^{3 / 2}\right)+\text { const. }
$$

Note

$$
|h(y)|=0\left(y^{-2 / 3}\right)+0\left(y^{-1 / 3-2 \varepsilon / 3}\right)=0\left(y^{-1 / 3-\delta}\right) \quad \delta>0
$$

We now estimate $\operatorname{Re}\left(i \int_{0}^{x} V_{\lambda}(-s) \frac{d s}{\sqrt{s}}\right)$.

Note that integrating by parts gives

$$
\int_{0}^{x} V_{\lambda}(-s) \frac{d s}{\sqrt{s}}=\frac{1}{\sqrt{x}} \int_{0}^{x} V_{\lambda}(-s) d s+\frac{1}{2} \int_{0}^{x}\left[\int_{0}^{t} V_{\lambda}(-s) d s\right] t^{-3 / 2} d t
$$


and that by Cauchy's integral theorem

$$
\int_{0}^{x} V_{\lambda}(-s) d s=\int_{0}^{x} V_{\operatorname{Re} \lambda}(-s) d s-\int_{\operatorname{Re} \lambda-x}^{\lambda-x} V(z) d z+\int_{\operatorname{Re} \lambda}^{\lambda} V(z) d z
$$

and thus

$$
\left|\operatorname{Im} \int_{0}^{x} V_{\lambda}(-s) d s\right| \leqq\left|\int_{\operatorname{Re} \lambda-x}^{\lambda-x} V(z) d z\right|+\left|\int_{\operatorname{Re} \lambda}^{\lambda} V(z) d z\right| \leqq c(1+|x|)^{1 / 2-\varepsilon}
$$

Thus by Eq. (A.8)

$$
\left|\operatorname{Re}\left(i \int_{0}^{x} V_{\lambda}(-s) \frac{d s}{\sqrt{s}}\right)\right| \leqq \text { const }
$$

Because of (A.9) and (A.7)

$$
\left|G_{1}(x)\right| \leqq \text { const } x^{-1} \exp \left(2 \operatorname{Im} z x^{1 / 2}\right)
$$

while by Eq. (A.5), for some $\delta_{1}>0$

$$
\begin{aligned}
& \left|\int_{1}^{x^{3 / 2}} N(y) h(y) y^{-1 / 3} d y\right| \leqq \text { const } x^{-1} \exp \left(2 \operatorname{Im} z x^{1 / 2}\right) \\
& + \text { const } \int_{1}^{x^{3 / 2}} \exp \left(2 \operatorname{Im} z y^{1 / 3}\right)\left(y^{-1-\delta_{1}}+\left|V_{\lambda}^{\prime}\left(-y^{2 / 3}\right) y^{-4 / 3}\right|\right) d y .
\end{aligned}
$$

Using Eq. (2.8) we have $\left|V_{\lambda}^{\prime}\left(-y^{2 / 3}\right) y^{-4 / 3}\right|=0\left(y^{-1-\delta_{2}}\right)$ for some $\delta_{2}>0$.

On the other hand an easy estimate gives

$$
\int_{1}^{x^{3 / 2}} \exp \left(2 \operatorname{Im} z y^{1 / 3}\right) y^{-1-\delta} d y \leqq \text { const } x^{-1 / 2(1+3 \delta)} \exp \left\{(2 \operatorname{Im} z) x^{1 / 2}\right\}
$$

so that

$$
\left|\int_{1}^{x^{3 / 2}} N(y) h(y) y^{-1 / 3} d y\right| \leqq \text { const } x^{-1 / 2-\delta_{3}} \exp \left(2 \operatorname{Im} z x^{1 / 2}\right)
$$

for some $\delta_{3}>0$. Going back to Eq. (A.2) we have

$$
|\alpha(x)| \leqq d_{1} x^{-1 / 2} \exp \left(2 \operatorname{Im} z x^{1 / 2}\right) \leqq d_{2}|\alpha(x)|
$$

for some $d_{1}, d_{2}>0$. This gives (ii) of the lemma.

Proof of Proposition A.2 We must show that neither $\phi_{\gamma}^{+}$nor $\phi_{\gamma}^{-}$is in $L^{2}$. Since they are linearly independent (we have $\left.W_{\gamma}(z, \lambda) \neq 0\right), \phi_{\gamma}^{+}(z, \lambda ; x)=\alpha_{1} \phi_{\gamma}^{-}(z, \lambda ; x)+$ $\beta_{1} \psi_{\gamma}^{-}(z, \lambda ; x)$ if $x<-1$, where $\beta_{1} \neq 0$. Similarly $\phi_{\gamma}^{-}(z, \lambda ; x)=\alpha_{2} \phi_{\gamma}^{+}(z, \lambda ; x)+$ $\beta_{2} \psi_{\gamma}^{+}(z, \lambda ; x)$ if $x>1$, where $\beta_{2} \neq 0$. The lower bounds of Lemma A.3 show that neither is in $L^{2}$.

Proof of Proposition A.1. We drop the subscripts $\gamma, z, \lambda$ and only mention the dependence of constants on $(z, \lambda)$ when uniform boundedness in $\Lambda$ is not obvious. We use the notation $\theta(x)$ for the indicator function of $[0, \infty)$. 
Let (see Eq. (2.4))

$$
G(x, y)=\phi^{+}\left(x_{>}\right) \phi^{-}\left(x_{<}\right) .
$$

Since $G$ is symmetric, it is enough to prove that $\theta(x-y) G(x, y)$ is the kernel of a bounded operator and since $\int|G|^{2} \theta(x+1) \theta(1-y) d x d y \leqq$ const., it is enough to consider the kernels $\theta(y-1) \theta(x-y) \phi^{+}(x) \phi^{-}(y)$ and $\theta(-x-1) \theta(x-y) \phi^{+}(x) \phi^{-}(y)$.

For $y \geqq 1, \phi^{-}(y)=\alpha \phi^{+}(y)+\beta \psi^{+}(y)$ and for $x \leqq-1, \phi^{+}(x)=\alpha^{\prime} \phi^{-}(x)+$ $\beta^{\prime} \psi^{-}(x)$. The constants $\alpha, \beta, \alpha^{\prime}, \beta^{\prime}$ are uniformly bounded in $\Lambda$ because the Wronskian of $\phi^{+}$and $\psi^{+}$(and of $\phi^{-}$and $\psi^{-}$) is 1. Since $\phi^{+} \in L^{2}([1, \infty)$ ) and $\phi^{-} \in L^{2}((-\infty,-1])$ we need only prove boundedness of the integral operators with kernels

a) $\theta(x-y) \theta(y-1) \phi^{+}(x) \psi^{+}(y)=K_{1}(x, y)$

and

b) $\theta(x-y) \theta(-x-1) \psi^{-}(x) \phi^{-}(y)=K_{2}(x, y)$.

We use the Holmgren-Schur estimate that shows the operator norms are bounded by

$$
\left(\sup _{u} \int\left|K_{j}(x, y)\right| d y\right)^{1 / 2}\left(\sup _{y} \int\left|K_{j}(x, y)\right| d x\right)^{1 / 2}
$$

Consider a) first. We let

$$
I_{1}(x)=\int\left|K_{1}(x, y)\right| d y, \quad I_{2}(y)=\int\left|K_{1}(x, y)\right| d x .
$$

We have

$$
\begin{aligned}
I_{1}(x) & =\left|\phi^{+}(x)\right| \int_{1}^{x}\left|\psi^{+}(y)\right| d y & & x \geqq 1 \\
& =0 & & x<1 .
\end{aligned}
$$

Using the estimates of Lemma A.2 we have

$$
\int_{1}^{x}\left|\psi^{+}(y)\right| d y \leqq \text { const } \int_{1}^{x} y^{-1 / 2} \exp \left\{\frac{2}{3} y^{3 / 2}-\operatorname{Re} z y^{1 / 2}+\frac{1}{2} \operatorname{Re} \int_{0}^{y} V_{\lambda}(t) \frac{d t}{\sqrt{t}}\right\} d y .
$$

Integrating by parts in the same way as in the proof of Lemma A.2 gives

$$
\int_{1}^{x}\left|\psi^{+}(y)\right| d y \leqq \text { const } x^{-1} \exp \left\{\frac{2}{3} x^{3 / 2}-\operatorname{Re} z x^{1 / 2}+\frac{1}{2} \operatorname{Re} \int_{0}^{x} V_{\lambda}(t) \frac{d t}{\sqrt{t}}\right\}
$$

and thus $I_{1}(x) \leqq$ const. $x^{-1}$.

We have

$$
\begin{aligned}
I_{2}(y) & =\int_{y}^{\infty}\left|\phi^{+}(x)\right| d x\left|\psi^{+}(y)\right| & & y \geqq 1 \\
& =0 & & y<1 .
\end{aligned}
$$


A similar integration by parts gives

$$
I_{2}(y) \leqq \text { const } y^{-1} .
$$

Thus the operator given by $K_{1}$ is uniformly bounded in $\Lambda$.

In case b) let

$$
J_{1}(x)=\int\left|K_{2}(x, y)\right| d y, \quad J_{2}(y)=\int\left|K_{2}(x, y)\right| d x
$$

and note that

$$
\begin{aligned}
J_{1}(x) & =\left|\psi^{-}(x)\right| \int_{-\infty}^{x}\left|\phi^{-}(y)\right| d y & & x \leqq-1 \\
& =0 & & x>-1 .
\end{aligned}
$$

Thus

$$
\begin{aligned}
J_{1}(-x) & \leqq \text { const } x^{-1 / 2} \exp \left\{(\operatorname{Im} z) x^{1 / 2}\right\} \int_{x}^{\infty} \exp \left(-\operatorname{Im} z y^{1 / 2}\right) d y \quad x \geqq 1 \\
& \leqq \text { const }
\end{aligned}
$$

while

$$
\begin{aligned}
J_{2}(y) & =\left|\phi^{-}(y)\right| \int_{y}^{-1}\left|\psi^{-}(x)\right| d x & & y \leqq-1 \\
& =0 & & y>-1
\end{aligned}
$$

and thus

$$
\begin{aligned}
J_{2}(-y) & \leqq \text { const } \exp \left(-\operatorname{Im} z y^{1 / 2}\right) \int_{1}^{y} x^{-1 / 2} \exp \left\{(\operatorname{Im} z) x^{1 / 2}\right\} d x \quad y \geqq 1 \\
& \leqq \text { const. }
\end{aligned}
$$

Hence the operator given by $K_{2}$ is uniformly bounded in $\Lambda$.

This completes the proof of Proposition A.1.

\section{References}

1. Anguilar, J., Combes, J. M.: Commun. Math. Phys. 22, 269-279 (1971)

2. Anderson, P. W. : Phys. Rev. 109, 1492 (1958)

3. Avron, J. E. : Phys. Rev. Lett. 37, 1568 (1976); J. Phys. A. 12, 2393 (1979)

4. Avron, J. E., Herbst, I. W. : Commun. Math. Phys. 52, 239-254 (1977)

5. Balslev, E., Combes, J. M.: Commun. Math. Phys. 22, 280-294 (1971)

6. Bentosela, F. : Commun. Math. Phys. 68, 173-182 (1979)

7. Faris, W. G., Lavine, R. : Commun. Math. Phys. 35, 39-48 (1974)

8. Feller, W. : An introduction to probability theory and its applications v. II (2nd ed) New York: Wiley 1971

9. Goldstein, I. Ja., Molchanov, S. A. : Dokl. Akad. Nauk. SSSR 230, 761-764 (1976)

10. Goldstein, I. Ja., Molchanov, S. A., Pastur, L. A.: Funkts. Anal. Prilozhen. 11, 1-10 (1977)

11. Reed, M., Simon, B. : Methods of modern mathematical physics Vol. I. New York: Academic Press 1972

12. Rejto, P. A., Sinha, K.: Helv. Phys. Acta. 49, 389-413 (1976) 
13. Steinberg, S.: Arch. Rat Mech. Anal. 31, 372-380 (1968)

14. Thouless, D. J.: Phys. Rep. 13C, 95 (1974)

15. Titchmarsh, E. C.: Proc. R. Soc. A207, 321-328 (1951); A210, 30-47 (1951); J. Anal. Math. 4, 187-208 (1954/56)

16. Wannier, G. H.: Phys. Rev. 117, 432 (1960); 181, 1364 (1969); Rev. Mod. Phys. 34, 645 (1962)

17. Zak, J.: Phys. Rev. Lett. 20, 1477 (1968); Phys. Rev. 181, 1366 (1969)

18. Howland, J. S. : Proceeding of the special session on Mathematical Physics. AMS Meeting, Boulder, Co. April, 1980

Communicated by B. Simon

Received June 13, 1980; in revised form September 26, 1980 